\title{
Model-based variables for the kinematic assessment of upper-extremity impairments in post-stroke patients
}

\author{
Alessandro Panarese ${ }^{1 * \dagger}$, Elvira Pirondini ${ }^{2^{*}+}$, Peppino Tropea ${ }^{1}$, Benedetta Cesquii ${ }^{3,4}$, Federico Posteraro 5,6 \\ and Silvestro Micera ${ }^{1,2}$
}

\begin{abstract}
Background: Common scales for clinical evaluation of post-stroke upper-limb motor recovery are often complemented with kinematic parameters extracted from movement trajectories. However, there is no a general consensus on which parameters to use. Moreover, the selected variables may be redundant and highly correlated or, conversely, may incompletely sample the kinematic information from the trajectories. Here we sought to identify a set of clinically useful variables for an exhaustive but yet economical kinematic characterization of upper limb movements performed by post-stroke hemiparetic subjects.
\end{abstract}

Methods: For this purpose, we pursued a top-down model-driven approach, seeking which kinematic parameters were pivotal for a computational model to generate trajectories of point-to-point planar movements similar to those made by post-stroke subjects at different levels of impairment.

Results: The set of kinematic variables used in the model allowed for the generation of trajectories significantly similar to those of either sub-acute or chronic post-stroke patients at different time points during the therapy. Simulated trajectories also correctly reproduced many kinematic features of real movements, as assessed by an extensive set of kinematic metrics computed on both real and simulated curves. When inspected for redundancy, we found that variations in the variables used in the model were explained by three different underlying and unobserved factors related to movement efficiency, speed, and accuracy, possibly revealing different working mechanisms of recovery.

Conclusion: This study identified a set of measures capable of extensively characterizing the kinematics of upper limb movements performed by post-stroke subjects and of tracking changes of different motor improvement aspects throughout the rehabilitation process.

Keywords: Stroke, Robotic rehabilitation, Kinematics, Modeling

Abbreviations: FA, Factor analysis; FMA, Fugl-meyer assessment; ICA, Independent component analysis; MAS, Modified ashworth scale; MI, Motricity index; PCA, Principal component analysis

\footnotetext{
*Correspondence: a.panarese@sssup.it; elvira.pirondini@epfl.ch

${ }^{\dagger}$ Equal contributors

${ }^{1}$ The BioRobotics Institute, Scuola Superiore Sant'Anna, Viale R. Piaggio 34, 56025 Pontedera, Pisa, Italy

${ }^{2}$ Bertarelli Foundation Chair in Translational Neuroengineering, Center for Neuroprosthetics and Institute of Bioengineering, School of Engineering, École Polytechnique Fédérale de Lausanne (EPFL), Lausanne, Switzerland Full list of author information is available at the end of the article
} 


\section{Background}

Upper limb functions are altered in about $80 \%$ of acute stroke survivors and in about $50 \%$ of chronic post-stroke patients [1]. With the increasing of life expectancy, it has been estimated that stroke related impairments will be ranked to the fourth most important causes of adult disability in 2030 [2], prompting the need to design more effective diagnostic and rehabilitative tools [3, 4].

Together with more traditional and widely accepted clinical scales in the last two decades investigators have characterized post-stroke motor recovery also in terms of kinematic parameters extracted from hand and arm task-oriented movements [3, 5], which offer more objective measures of motor performance [6]. Indeed, clinical scales, whose reliability has often been questioned [7-9], may not be sensitive to small and more specific changes [10] and could be of limited use to distinguish different aspects of motor improvement $[11,12]$.

Previous robot-assisted clinical and pilot studies have proposed a large set of kinematic parameters to characterize motor improvements $[5,6,11]$. A few of them focused on finding a significant relationship between robotic measures collected longitudinally in post-stroke patients and clinical outcome measures, to increase acceptance of kinematic evaluation scales in practice $[5,6]$. Too little effort, however, has been made to identify the different aspects of movement improvement, how they can be described by kinematic robot-based measures [11], and whether they may dissociate with respect to recovery time course and to training response [11].

Indeed the range of potential changes in limb trajectory during recovery is not known a priori [12] and might not be fully represented by a set of arbitrarily selected parameters extracted from limb trajectories, even if the parameters were chosen according to a certain number of study hypotheses or to significant relationships with clinical scales. Moreover, these variables can be highly correlated and, thus, redundant. Although redundancy can be tackled by data reduction algorithms, such as Principal Component Analysis (PCA) or Independent Component Analysis (ICA) [5, 6], incomplete representation of information might still remain an overlooked issue.

In the present study we aimed at devising a novel method for identifying a set of kinematic measures potentially capable of fully highlighting and tracking changes of different aspects of movement performance throughout the rehabilitation training. Instead of starting from a certain number of a priori hypotheses, we sought to find which variables were essential for modeling trajectories of post-stroke patients and were, thus, informative of kinematic features of upper limb movements. We then tested whether the identified kinematic parameters $i$ ) were capable of highlighting changes in movement performance, ii) were to some extent redundant, and iii) were informative of different factors of post-stroke motor impairment, such as paresis, loss of fractionated movement and somatosensation, and abnormal muscle tone [13].

\section{Methods \\ Participants}

The data from 12 patients (6 men 6 women) were included in this study. All patients experienced a single unilateral cerebrovascular accident. 6 patients (sub-acute, age $71.8 \pm 5.4$ years) were enrolled less than 40 days after stroke, and 6 patients (chronic, age $64.0 \pm 12.9$ years) between 5 and 142 months post-stroke. Participants' FuglMeyer Assessment (FMA) scores [14, 15] were $20.5 \pm 9.0$ and $27.5 \pm 8.7$ for sub-acute and chronic patients, respectively. Table 1 reports a summary of the features related to all patients at the beginning of the therapy. Data related to the sub-acute patients and details on the rehabilitation protocol were already reported in a previous study [16].

Due to the limited sample size the inhomogeneity in the patient groups and in the treatment schedules (see Rehabilitation protocol), motor performances will not be compared between the two stroke survivor populations.

Seven neurologically intact age-matched subjects (5 men 2 women; age $72 \pm 5$ years) were also included in the study as control group (part of their data were published earlier [17]). Healthy participants exhibited normal ranges of motion and muscle strength and they did not show any functional disability.

\section{Rehabilitation protocol}

The patients and the healthy subjects were instructed to make point-to-point reaching movements forward and backward from the center of the workspace to one of eight different targets equally spaced around a circle of $14 \mathrm{~cm}$ of radius (Fig. 1 step 1) assisted by InMotion2 (Interactive Motion Technologies, Inc. Cambridge, Massachusetts) [18]. When the subjects sequentially carried out all the 16 movements, they completed one full turn.

The rehabilitation protocol consisted of $45 \mathrm{~min}$ of robot-assisted therapy (at least 65 turns per sessions) five days per week. Sub-acute patients practiced for 6 weeks whereas chronic patients for 4 weeks. The difference in the treatment schedules was due to the organizational structure of the hospital in which the treatment was carried out. The robot provided assisting force when patients were not able to reach the targets, except for $2 / 3$ turns per session (i.e., assessing turns) that were used to assess subjects' motor performance. In this study, only the data related to these assessing turns and the data recorded without application of external force fields for the healthy subjects were included. The protocol was approved by the Local Ethical Committee (Comitato Etico 
Table 1 Summary of stroke patients inserted in the study

\begin{tabular}{|c|c|c|c|c|c|c|c|}
\hline $\begin{array}{l}\text { Sub-Acute } \\
\text { Patients }\end{array}$ & Gender & Age yrs & $\begin{array}{l}\text { Days elapsed from } \\
\text { the accident }\end{array}$ & Dominance & Paretic Side & Stroke type & Location \\
\hline Sub01 & M & 82 & 37 & $\mathrm{R}$ & L & I & Right cortical-subcortical frontal \\
\hline Sub02 & F & 66 & 29 & R & L & । & Right Frontal-temporal-parietal \\
\hline Sub03 & M & 70 & 27 & $\mathrm{R}$ & L & । & Right cortical-subcortical precentral \\
\hline Sub04 & M & 70 & 24 & R & R & $\mathrm{H}$ & Left internal capsule \\
\hline Sub05 & M & 72 & 14 & $\mathrm{R}$ & L & । & Right cortical-subcortical parietal \\
\hline Sub06 & $\mathrm{F}$ & 71 & 19 & $\mathrm{R}$ & L & । & Right paramedian pontis \\
\hline $\begin{array}{l}\text { Chronic } \\
\text { patients }\end{array}$ & Gender & Age yrs & $\begin{array}{l}\text { Months elapsed from } \\
\text { the accident }\end{array}$ & Dominance & Paretic Side & Stroke type & Location \\
\hline Sub07 & $\mathrm{F}$ & 58 & 143 & $\mathrm{R}$ & L & I & Right talamus \\
\hline Sub08 & $\mathrm{F}$ & 67 & 139 & $\mathrm{R}$ & L & $\mathrm{H}$ & Right talamus \\
\hline Sub09 & M & 47 & 5 & $\mathrm{R}$ & R & $\mathrm{H}$ & Left nucleus lenticolar \\
\hline Sub10 & $\mathrm{F}$ & 61 & 5 & $\mathrm{R}$ & L & $\mathrm{H}$ & Right thalamus capsular \\
\hline Sub11 & $\mathrm{F}$ & 86 & 58 & $\mathrm{R}$ & R & । & Left nucleus caudatus and thalamus \\
\hline Sub12 & M & 65 & 8 & R & L & $\mathrm{H}$ & Right fronto-parietal \\
\hline
\end{tabular}

Labels in the $2^{\text {nd }}, 5^{\text {th }}, 6^{\text {th }}$, and $7^{\text {th }}$ columns refer to: $F$ Female, $M$ Male, $L$ Left, $R$ Right, $H$ Hemorrhagic, $I$ Ischemic

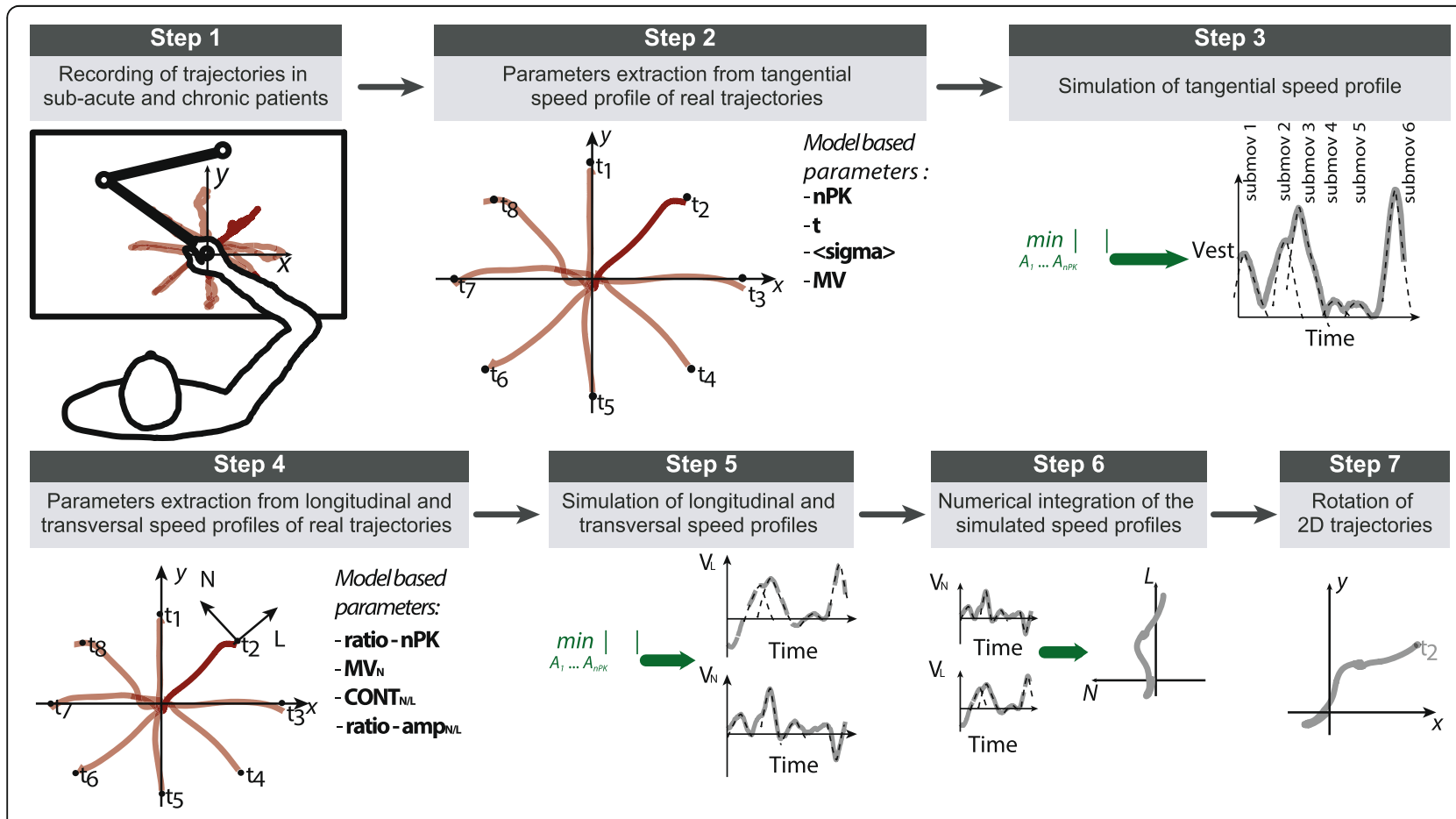

Fig. 1 Schematic overview of the computational model for post-stroke trajectories simulation. (1) Endpoint kinematics of one pathological subject making point to point forward and backward movements from the center of the workspace to one of eight different targets equally spaced around a circle of $14 \mathrm{~cm}$ of radius assisted by InMotion2. (2) Kinematic parameters are extracted from the real trajectories of the post-stroke subjects. The tangential speed profile of real trajectories (for each movement direction and subject, separately for each group of patients and time of therapy) is analyzed to extract probability distributions of $\mathbf{n P K},\langle\boldsymbol{\sigma}\rangle, \mathbf{T}$, and $\mathbf{M V}$. (3) Based on the inferred probability distributions, tangential speed profiles of simulated trajectories are generated by solving a constrained optimization problem (Eq. 2). (4) The transversal and longitudinal speed profiles of real trajectories are analyzed to extract probability distributions of ratio-amp $\mathbf{L}_{\mathrm{L}}$, ratio-amp $\mathbf{N}$, ratio-nPK, $\mathbf{M V}_{\mathbf{N}}, \mathbf{C O N T}_{\mathrm{L}}$, and $\mathbf{C O N T}_{\mathbf{N}}$. (5) From the simulated tangential velocities and the inferred distributions of kinematic parameters, transversal and longitudinal speed profiles of simulated trajectories are generated, by solving an unconstrained optimization problem (Eq. 4). (6) The trajectories in the Cartesian space defined by the (L, N) axes (see step 4) are obtained from the speed profiles by numerical integration. (7) The generated trajectories are then rotated by means of a geometrical transformation to reproduce the point-to-point movements performed by post-stroke subjects during a turn in the InMotion2 coordinate frame system 
Azienda Ospedaliera Universitaria di Pisa. Reference $\mathrm{N}^{\circ}$ : 2754, year 2009).

Participants' upper limb motor functions were evaluated before $\left(T_{0}\right)$ and after $\left(T_{1}\right)$ the rehabilitative treatment by an experienced physiatrist using clinical scales: FMA [14, 15], Modified Ashworth Scale (MAS) for shoulder and elbow [19], and Motricity index (MI) [20].

\section{Computational model}

We devised a computational model able to simulate end-point planar trajectories of stroke patients for the given upper-limb rehabilitation protocol (see Rehabilitation protocol) starting from the knowledge of a limited number of kinematic parameters (i.e., Model-based parameters), whose values were specified according to the experimental data (i.e., extracted from the real trajectories of the post-stroke patients included in this study). Indeed, simulated trajectories geometrically similar to those of stroke patients, and with comparable kinematic features, would imply that the Model-based parameters are a thorough and yet parsimonious way to represent the kinematic information contained in the trajectories of post-stroke subjects.

Unlike previous studies [21, 22], point-to-point movements were modeled specifying the complete trajectory on the plane. Indeed, unidimensional models are suitable for modeling healthy adult movements, which are approximately straight in the Cartesian space [21, 23], but inevitably they neglect to characterize the deviations from the theoretical path (i.e., the straight line connecting the start and the target points), which are indeed important markers of motor improvements of post-stroke patients.

We started by modeling the time profile of a trajectory's tangential speed $v_{T}(t)$, as a sum of submovement curves (i.e., single discrete movements that contribute to the resulting movement path, Fig. 1, step 3):

$$
v_{T}(t)=\sum_{i=1}^{\mathbf{n P K}} A_{i} e^{-\left(\frac{t-t_{i}}{\sqrt{2} \sigma_{i}}\right)^{2}}
$$

such that:

$$
\begin{aligned}
& 0 \leq t \leq \boldsymbol{t}, \\
& \frac{1}{\boldsymbol{t}} \int_{0}^{\boldsymbol{t}} v(t) d t=M V, \\
& E[\sigma]=\langle\boldsymbol{\sigma}>.
\end{aligned}
$$

These curves were assumed to be bell-shaped Gaussian profiles centered at time $t_{i}$ with amplitude $\boldsymbol{A}_{\boldsymbol{i} i}$ and duration proportional to $\sigma_{i}$. Indeed, Krebs et al. [24] demonstrated that submovement speed profiles are remarkably similar across patients even though neurological damages are not, and can be appropriately approximated by nearly symmetrical $\beta$-functions or by Gaussian curves. The peak times, $t_{i}$, in the tangential speed profile were randomly drawn from a uniform distribution $U(0, \mathbf{t})$, with the constraint of being spaced at least by $2<\sigma>$ to avoid peak overlapping, and $\sigma_{i}$ were randomly chosen such that $E[\sigma]$ $=\langle\boldsymbol{\sigma}\rangle$.

This $v_{T}(t)$ profile model requires the specification of the number of submovements of which the entire movement is composed of i.e., $n=-\mathbf{n P K}$, their average peak duration $\langle\boldsymbol{\sigma}\rangle$, the movement duration, $\mathbf{t}$, and the average tangential speed, MV. To generate simulated $v_{T}(t)$ profiles specific for each patient group (sub-acute, chronic) and time point during the therapy $\left(T_{0}, T_{1}\right)$, we first extracted the values of $\mathbf{n P K},\langle\boldsymbol{\sigma}\rangle, \mathbf{t}, \mathbf{M V}$ from the trajectories recorded during the experiments (Fig. 1, step 2). In particular, nPK was obtained with the constraint that peaks amplitude had to be larger than the $10 \%$ of the maximum speed amplitude [25] and $\langle\boldsymbol{\sigma}\rangle$ was computed as the average $\sigma$ of the Gaussian curves fitted to each tangential submovement.

We then estimated different (for each patient group and time point) probability distributions for these parameters (pooling data across repetitions subjects, and movement directions). A new random sample from each distribution was drawn each time a new simulated tangential speed profile had to be generated. The new simulated tangential speed profile was computed by solving a numerical optimization problem (constrained problem with the active set algorithm implemented in the function fmincon) in Matlab (Mathworks, Inc., MA, USA):

$$
\min _{A_{1} \ldots A_{\mathbf{n P K}}}\left|M V-\frac{1}{\mathbf{t}} \int_{0}^{t} v\left(t, A_{1} \ldots A_{\mathbf{n P K}}\right) d t\right|
$$

subject to the constraint:

$$
A_{i}>0, \quad i=1, \ldots, \mathbf{n P K}
$$

The problem of generating simulated two-dimensional trajectories required the specification of end-point motion in two axes on the plane of movement. We chose the axis defined by the theoretical path and the corresponding orthogonal axis (the $\mathrm{L}$ and $\mathrm{N}$ axes respectively, in Fig. 1, step 4). The end-point motion was specified in terms of the projections of the velocity vector, $\boldsymbol{v}(t)$, along these two axes: the longitudinal, $v_{L}(t)$, and the transversal, $v_{N}(t)$, speed profiles. This problem cannot be solved analytically because $v_{T}(t)$ did not contain information regarding the direction of the movement, thus a number of assumptions were made to obtain approximated solutions for $v_{L}(t)$ and $v_{N}(t)$.

First similar to $v_{T}(t)$, we assumed that $v_{L}(t)$ and $v_{N}(t)$ were composed of a sum of Gaussian bell-shaped curves. Indeed, these curves represent submovements projected onto orthogonal directions: the longitudinal, L, and the transversal, N, axes (see Fig. 1, step 4). Moreover, the correlation between a fitted sum of Gaussian bellshaped curves with the experimental longitudinal and 
transversal speed profiles was high (Pearson's correlation: $\rho=0.75 \pm 0.18$ ).

Second we considered that a number of peaks in $v_{T}(t)$ could be the result of movements traveling predominantly in the longitudinal or transversal direction. To take this into account, two additional parameters were added to the model: $\mathbf{C O N T}_{\mathbf{L}}$ and $\mathbf{C O N T}_{\mathbf{N}}$, i.e. the number of peaks in $v_{L}(t)$ and $v_{N}(t)$, respectively, producing a corresponding peak in $v_{T}(t)$. $\mathbf{C O N T}_{\mathbf{x}}$ (with $x \in\{L, N\}$ ) was calculated on the longitudinal or the transversal speed profile of each experimental trajectory as the sum of "contributing peaks", i.e. peaks falling within a time interval as large as $\boldsymbol{\sigma}$ and centered on peaks of the tangential velocity. For $v_{L}(t)$ and $v_{N}(t)$, the number of peaks (i.e., $\mathbf{n P K} \mathbf{L}_{\mathbf{L}}$ and $\mathbf{n} \mathbf{P K} \mathbf{N}_{\mathbf{N}}$, respectively) were calculated following the same technique used for $v_{T}(t)$ considering separately the positive and the negative part of the speed profile, resulting in the estimation of positive and negative peaks, respectively.

Third $\mathbf{n} \mathbf{P K} \mathbf{L}$ and $\mathbf{n} \mathbf{P K} \mathbf{N}_{\mathbf{N}}$ were assumed to be comparable because they had, on average, similar values in our dataset (except for sub-acute patients at $T_{0} ; p=0.04$ Wilcoxon rank sum test, significance level $\alpha=0.05$ ) and were computed from the ratio-nPK (ratio between the number of peaks in the longitudinal and in the tangential velocity profiles). However, should this assumption be falsified in a new cohort of patients larger than ours, the model could be easily modified to include both $\mathbf{n} \mathbf{P K} \mathbf{L}_{\mathbf{L}}$ and $\mathbf{n} \mathbf{P K} \mathbf{N}_{\mathbf{N}}$ in the Model-based parameters.

The longitudinal and transversal speed profiles can be expressed similarly to $v_{T}(t)$ (see Eq. 1 ) with $t_{i}^{(x)}$ and $A_{i i}^{(x)}$ the central instants and amplitudes of the peaks in the speed profile with $x \in\{L, N\}$. A number of $\mathbf{C O N T}_{\mathbf{x}}$ central instants were the same at which peaks in $v_{T}(t)$ occur, whereas the remaining $\mathbf{n} \mathbf{P K} \mathbf{x}_{\mathbf{x}}-\mathbf{C O N T}_{\mathbf{x}}$ were randomly chosen in $[0, \mathbf{t}]$, with a constraint on peak-to-peak distance similar to that for $v_{T}(t)$ :

$$
v_{x}(t)=\sum_{i=1}^{\mathbf{n P K}_{\mathbf{x}}} A_{i}^{(x)} e^{-\left(\frac{t-t_{i}(x)}{\sqrt{2} \sigma_{i}}\right)^{2}}
$$

such that:

$$
\begin{aligned}
& 0 \leq t \leq \boldsymbol{t}, \\
& \frac{1}{\boldsymbol{t}} \int_{0}^{t} v(t) d t=M V_{x}, \\
& E[\sigma]=\langle\boldsymbol{\sigma}\rangle .
\end{aligned}
$$

with $x \in\{L, N\}$. The computation of the two velocities can be reduced to a single unconstrained optimization problem to find the amplitudes $\left[A_{i}^{(L)} \ldots A_{\mathbf{n P K}_{\mathrm{L}}}^{(L)}, A_{i}^{(N)} \ldots A_{\mathbf{n P K}}^{(N)}\right]$ (see Fig. 1 step 5). Initial values for these amplitudes were set by using two additional parameters: ratio- $\mathbf{a m p}_{\mathbf{L}}$, the ratio between average longitudinal and average tangential peak amplitude, and ratio-amps (i.e., same parameter with the average transversal peak amplitude in the numerator). Specifically, the function to minimize was:

$$
\begin{aligned}
& \min _{\left[A_{1}^{(l)} \ldots A_{\mathbf{n F}_{\mathrm{L}}}^{(l)}, \mathrm{A}_{1}^{(n)} \ldots A_{\mathrm{nPK}_{\mathrm{N}}}^{(n)}\right]} \alpha\left|M V_{l}-\frac{1}{\mathbf{t}} \int_{0}^{t} v_{l}\left(t, A_{1}^{(l)} \ldots A_{\mathbf{n P K}_{\mathrm{L}}}^{(l)}\right) d t\right| \\
& +\beta\left|M V_{n}-\frac{1}{\mathbf{t}} \int_{0}^{t} v_{n}\left(t, A_{1}^{(n)} \ldots A_{\mathbf{n P K}}^{(n)}\right) d t\right|
\end{aligned}
$$

with $\alpha$ and $\beta$ two parameters $(\alpha, \beta \leq 1)$ whose values were chosen to obtain a solution $v=\left(v_{L}(t), v_{N}(t)\right)$ meeting the requirements for $\mathrm{MV}_{\mathrm{L}}$ and $\mathrm{MV}_{\mathrm{N}}$ (i.e., average $v_{L}(t)$ and $v_{N}(t)$, respectively) at different compliance levels. In our simulations we used $\alpha=0.8$ and $\beta=0.2$.

The generation of $v_{L}(t)$ and $v_{N}(t)$ starting from the peaks (timing and amplitude) in the tangential velocity (that indicate changes in 2D movement direction and then determine 2D shape) allows the model to preserve the link between longitudinal and transversal speed components which was present also in the real trajectories (see Additional file 1: Figure S1 for a comparison of correlation values between longitudinal and transversal components in simulated and real trajectories).

As for the tangential speed the values of the six parameters required for the generation of simulated $v_{L}(t)$ and $v_{N}(t)\left(\mathbf{M V}_{\mathbf{N}}, \mathbf{C O N T}_{\mathbf{L}}, \mathbf{C O N T}_{\mathbf{N}}\right.$, ratio-amp $\mathbf{L}$, ratio$\mathbf{a m p}_{\mathbf{N}}$,ratio-nPK) were extracted from the trajectories recorded during the experiments, and subsequently pooled to estimate probability distributions, which were specific for each patient group and time point during the therapy (Fig. 1, step 2 and step 4). $\mathbf{M V}_{\mathbf{L}}$ was not directly estimated from the experimental trajectories but calculated as the ratio between the distance center-target, $\mathbf{D}=14 \mathrm{~cm}$, and the overall (experimental) duration of the movement, $\mathbf{t}$.

Once the speed profiles were computed (by solving an unconstrained optimization problem with the active set algorithm implemented in the function fminsearch of Matlab) they were numerically integrated over time to obtain the trajectory components along the longitudinal and transversal directions: $x_{L}(t)$ and $x_{N}(t)$, respectively (see Fig. 1, step 6). Finally, the two trajectory components were rotated to express the simulated end-point trajectory in the InMotion2 coordinate frame (see Fig. 1, step 7).

Sensitivity analysis was performed to demonstrate the stability of the model: for both group of patients and time points $\left(T_{0}\right.$ and $\left.T_{1}\right)$ a parameter at a time was varied (ten variations equally distributed across its probability distribution) while keeping the other parameters fixed at the average value of their probability distribution. Results of sensitivity analysis were comparable to those found with the parameters values chosen showing that the model was robust for variation of the parameters (see Additional file 2: Figure S2). 


\section{Model validation}

The mean Euclidean distance normalized by the total distance traveled during the reaching movement was used to assess the geometrical similarity between real and simulated trajectories $\boldsymbol{E}_{R S}$. Simulated trajectories were considered similar to the real ones if the range of variation of $\boldsymbol{E}_{R S}$ was comparable to $\boldsymbol{E}_{I}^{(R)}$, i.e., the intrinsic trajectory variability (inter-subject and intra-subject) of real curves.

We then validated the ability of the simulated trajectories to capture significant kinematic features of real movements by comparing the values of an extensive set of additional kinematic parameters not used by the model, computed on both simulated and real trajectories. This set of parameters was chosen to extensively characterize longitudinal and transversal speed profiles and to check the suitability of the optimization strategies used to simulate the speed profiles (i.e., tangential, longitudinal, and transversal) and, consequently, the trajectories. The following parameters (i.e., Evaluation parameters) were considered: $\mathbf{M V}_{\mathbf{L}}$, average longitudinal speed; $\mathbf{A}$ average amplitude of peaks in $\nu_{T}(t) ; \mathbf{A}_{\mathbf{L}}-$ pos and $\mathbf{A}_{\mathbf{L}}-\mathbf{n e g}\left(\mathbf{A}_{\mathbf{N}}-\right.$ pos and $\mathbf{A}_{\mathbf{N}}-$ neg) average amplitude of positive and negative peaks for $v_{L}(t)\left(v_{N}(t)\right) ; \mathbf{n} \mathbf{P K} \mathbf{L}_{\mathbf{L}}-\mathbf{p o s}$ and $\mathbf{n} \mathbf{P K} \mathbf{K}_{\mathbf{L}}-\mathbf{n e g}\left(\mathbf{n} \mathbf{P K} \mathbf{N}_{\mathbf{N}}-\mathbf{p o s}\right.$ and $\left.\mathbf{n P K} \mathbf{N}_{\mathbf{N}}-\mathbf{n e g}\right)$ the number of positive and negative peaks for $v_{L}(t)\left(v_{N}(t)\right) ; \mathbf{C O N T}_{\mathbf{N}}$-amp the ratio between the average transversal and tangential peak amplitudes, computed only for "contributing" peaks; $\mathbf{C O N T}_{\mathbf{L}}$-amp similar parameter for $v_{L}(t)$; MD the mean absolute value of the distance between the actual trajectory and the theoretical path; overlap the overlapping area between two submovements (intersection between the two Gaussian curves describing the submovements normalized by the sum of the two areas).

As a further validation of the model we tested the similarity between shoulder and elbow angular excursions computed from the simulated and the real trajectories using the two-link model for upper-limb movements introduced in [25]. Indeed, previous studies evaluated shoulder and elbow angular excursions during different tasks and reported an abnormal joint coupling in post-stroke patients that reduces during rehabilitative treatment [26-28].

Joint angles were estimated as:

$$
\begin{aligned}
\theta_{1} & =\tan ^{-1}(\mathrm{y}, \mathrm{x})-\tan ^{-1}\left(\mathrm{k}, \mathrm{x}^{2}+\mathrm{y}^{2}+\mathrm{l}_{1}^{2}-\mathrm{l}_{2}^{2}\right) \theta_{2} \\
& =\tan ^{-1}\left(\mathrm{k}, \mathrm{x}^{2}+\mathrm{y}^{2}-\mathrm{l}_{1}^{2}-\mathrm{l}_{2}^{2}\right)+\theta_{1}
\end{aligned}
$$

where $\theta_{1}$ and $\theta_{2}$ are respectively shoulder and elbow joint angles $\mathrm{k}=\sqrt{\left(\mathrm{x}^{2}+\mathrm{y}^{2}+\mathrm{l}_{1}^{2}+\mathrm{l}_{2}^{2}\right)^{2}-2\left[\left(\mathrm{x}^{2}+\mathrm{y}^{2}\right)^{2}+\mathrm{l}_{1}^{4}+\mathrm{l}_{2}^{4}\right]}$, $-\pi \leq \tan ^{-1} \leq \pi, l_{1}$ is the upper arm length, and $l_{2}$ the lower arm length. Values of the parameters $l_{1}$ and $l_{2}$ were estimated both for simulated and real trajectories from the measurements of the 50th percentile for U.S. males [29]: $\mathrm{l}_{1}$ was estimated as $0.282 \mathrm{~m}$; whereas $\mathrm{l}_{2}$ was calculated as the sum of forearm length $(0.254 \mathrm{~m})$ the distance from the wrist to the handle $(0.076 \mathrm{~m})$, and the handle robot radius $(0.03 \mathrm{~m})$.

Joint angular excursions computed from the simulated and the real trajectories were compared using $\boldsymbol{d}_{\%}$ (the average distance between two angular trajectories resampled to the same number of time points and normalized by the maximum angular excursion of the real trajectory).

\section{Investigation of underlying factors of motor recovery}

Finally we inspected the Model-based parameters both to evaluate redundancy among the parameters and to find whether longitudinal variations in these variables might have been explained by a restricted number of underlying (and unobserved) factors, putatively reflecting different aspects of motor recovery [11]. For this purpose, we performed a Factor Analysis (FA) with Maximum Likelihood extraction and promax rotation method on the Model-based parameters. These were pooled from all movement directions and repetitions for healthy subjects and patients at each day of rehabilitation. The number of retained factors was selected on the basis of "cleanliness of factor structure", and both the individual $(>5 \%)$ and the cumulative percentage $(\geq 70 \%)$ of total variance explained [30]. For each factor, the parameters with loadings $>0.6$ were clustered together [30]. Variables with loadings not exceeding the threshold for none of the factors were named "shared". To determine the time course of each factor along the rehabilitation process, for each patients group separately, we fitted the data to different functions: straight line, exponential decay, and double-exponential decay. The best fit was then selected among these three functions according to the Bayesian Information Criterion (BIC) [31].

\section{Statistical analyses}

Comparisons between $T_{O}$ and $T_{1}$ clinical scores and kinematic parameters were made with a Wilcoxon signed-rank test (significance level $\alpha=0.05$ ), for the two groups of patients separately. Parameters from real and simulated trajectories were analyzed separately. $\boldsymbol{E}_{R S}$ values were compared with the intrinsic variability of the real trajectories, $\boldsymbol{E}_{I}^{(R)}$, for both groups of patients and time points $\left(T_{O}\right.$ and $\left.T_{1}\right)$ using a Wilcoxon rank sum test (significance level, $\alpha=0.05$ ).

\section{Results}

Simulated trajectories reliably approximated real trajectories of post-stroke subjects

Simulated trajectories were generated for sub-acute and chronic patients both at time $T_{O}$ and $T_{1}$ (Fig. 2). As explained in Methods (Computational model) our aim was 


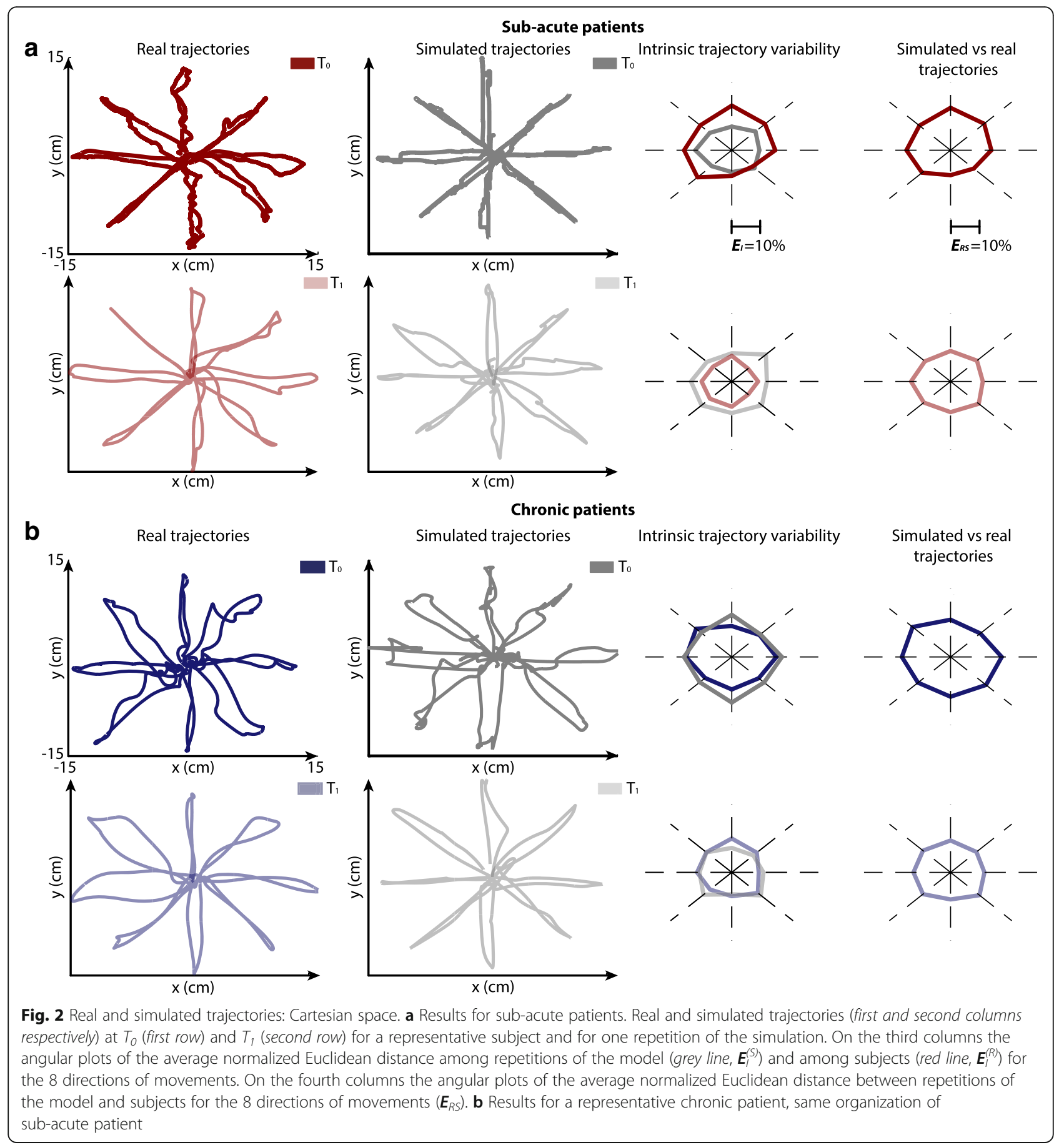

not to precisely reconstruct real trajectories, but to generate simulated trajectories consistent with the real ones. Indeed, simulated trajectories showed significant geometrical similarity with the real trajectories. In fact, the range of variation of $\boldsymbol{E}_{R S}$ was comparable to the intrinsic trajectory variability of real curves, $\boldsymbol{E}_{I}^{(R)}$, both at $T_{O}$ and $T_{1}$. The average $\boldsymbol{E}_{R S}$ (across movement directions and subjects) was $14.52 \pm 0.78 \%$ at $T_{0}$ and $13.02 \pm 0.52 \%$ at $T_{1}$, for sub-acute patients, and $16.88 \pm 1.43 \%$ at $T_{0}$ and
$13.05 \pm 1.25 \%$ at $T_{1}$, for chronic patients. Comparable ranges were confirmed by statistical tests. Indeed, only $\boldsymbol{E}_{R S}$ for sub-acute patients at $T_{1}$ was higher than $\boldsymbol{E}_{I}^{(R)}(p=0.008)$.

Interestingly $\boldsymbol{E}_{I}^{(R)}$ decreased after therapy, showing a reduction of performance variability within each group of patients, which was correctly captured by our model in the case of chronic patients $\left(\boldsymbol{E}_{I}^{(R)}: 15.71 \pm 2.87 \%\right.$ at $T_{0}$ and $9.90 \pm 2.43 \%$ at $T_{1}$, for sub-acute; $15.61 \pm 3.38 \%$ at 
$T_{O}$ and $11.87 \pm 2.18 \%$ at $T_{1}$ for chronic; $\boldsymbol{E}_{I}^{(S)}: 10.70 \pm$ $1.18 \%$ at $T_{O}$ and $13.88 \pm 1.05 \%$ at $T_{1}$ for sub-acute; $16.65 \pm 1.48 \%$ at $T_{O}$ and $11.71 \pm 2.60 \%$ at $T_{1}$ for chronic).

Finally the similarity between real and simulated angular excursions (elbow and shoulder) was assessed (see Fig. 3). The low $\boldsymbol{d}_{\%}$ values between simulated and real angular trajectories (19\% for the shoulder and $5 \%$ for the elbow, on average) showed that joint movements of post-stroke subjects were also finely reproduced.

Taken together these results demonstrated that the proposed computational model was able to simulate trajectories similar to those observed in post-stroke subjects at different impairment levels, both in the Cartesian and in the joint spaces.

Model-based parameters represents kinematic features of post-stroke trajectories

Although measuring motor improvements by means of clinical scales was not one of the main aims of this study we reported the scores of both sub-acute and chronic patients after therapy for the purpose of observing parallel significant improvements in both clinical scales and kinematic measures from point-to-point upper limb movements (Fig. 4a). The clinical scores partially correlated. In particular MAS score for the shoulder and the elbow correlated significantly $(r=0.85, p<0.001)$, as well as FMA score and Motricity index $(r=0.88, p<0.001)$. Moderate correlation between the ability to perform isolated joint movements (FMA score) and upper-limb strength (Motricity index) and, in contrast, low correlation with MAS score $(r=0.14, p>0.05)$, were in agreement with previous work $[6,32]$.

We quantified movements' improvements by computing both the Model-based and the Evaluation parameters on patients' trajectories (Fig. $4 \mathrm{~b}$ and Additional file 3: Figure S3).

Subjects' movement duration t, significantly diminished both for sub-acute and chronic patients across training sessions $(p<0.05)$. The movement smoothness (nPK) and $\mathbf{M} \mathbf{V}_{\mathbf{N}}$, instead, increased significantly along the treatment in both groups, whereas MV only in subacute patients $(p<0.05)$. Consistently with the increase of the mean velocity, a slight increment in longitudinal peaks amplitude (ratio-amp $\mathbf{a}$ ) and a significant decrease in transversal peaks amplitude (ratio- $\mathbf{a m p}_{\mathbf{N}}$ ) were

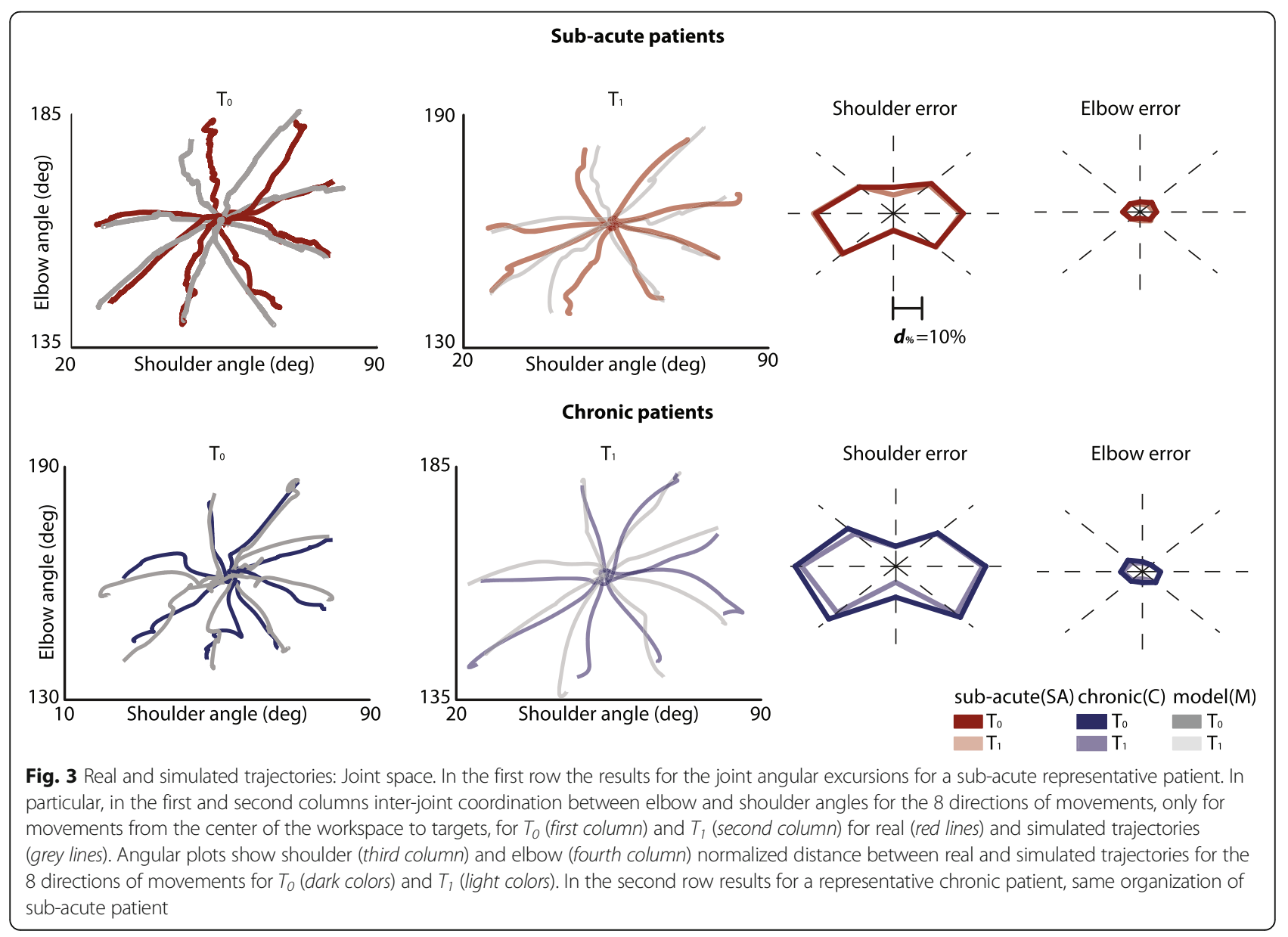




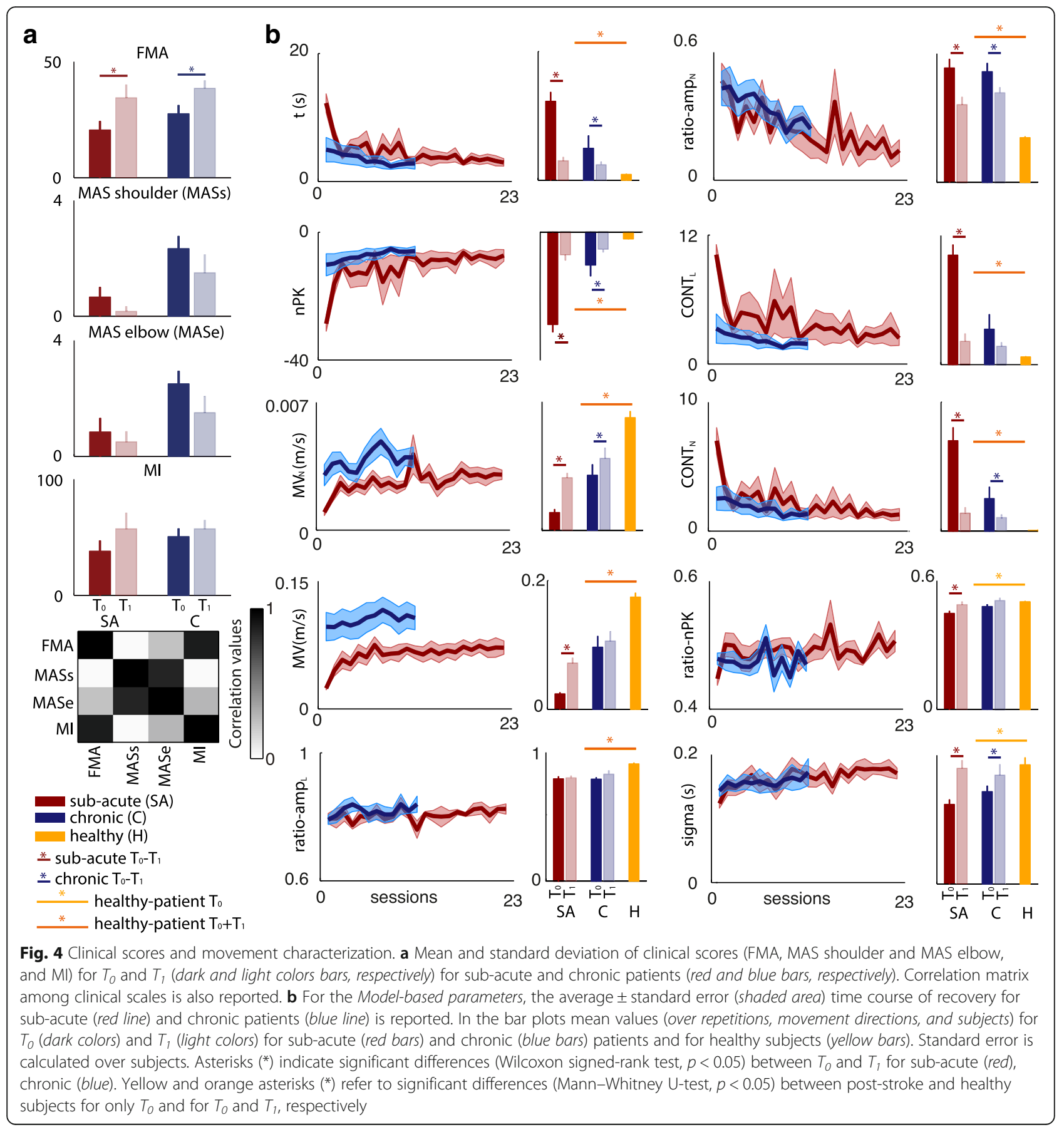

observed $(p<0.05)$, indicating a progressive translation of submovements toward the longitudinal direction. The increase in movement smoothness, instead, was reflected in a significant reduction of both longitudinal $\left(\mathrm{CONT}_{\mathbf{L}}\right.$, $p<0.05$ for sub-acute patients) and transversal peaks contribution $\left(\mathbf{C O N T}_{\mathbf{N}}, p<0.05\right.$ both for sub-acute and chronic patients). Interestingly, ratio-nPK had only a slight increase during the therapy, but it reached values comparable to those observed in the healthy subjects. Finally, the significant rise in both patient groups of the $\sigma$ values $(p<0.05)$ revealed an increment of submovements' duration, which, together with improved smoothness and increased overlap (Additional file 3: Figure S3), was consistent with the proposed mechanism of submovements blending during recovery [3]. All parameters values except ratio-nPK and $\langle\boldsymbol{\sigma}\rangle$ were significantly different $(p<0.003)$ between post-stroke and healthy subjects at $T_{0}$ and $T_{1}$, showing that albeit there were significant motor improvements in upper limb movements, the patients' recovery was not complete. 
We then investigated whether the kinematic features of real trajectories (and their evolution during the rehabilitation process) were well reproduced by simulated trajectories by comparing the values of the Evaluation parameters calculated on both trajectory types at $T_{O}$ and $T_{1}$ (Model-based parameters were used for the generation of the simulated trajectories as explained in section 2.3, and could not be used for the purpose of model evaluation). The Evaluation parameters were chosen because they were not directly linked to the Model-based parameters by means of known mathematical relationships. Indeed, only $\mathbf{n P K} \mathbf{K}_{\mathbf{x}}-\mathbf{p o s}$ and $\mathbf{n P K} \mathbf{K}_{\mathbf{x}}-\mathbf{n e g}$ (with $x \in\{L, N\}$ ) correlated with $\mathbf{n P K}$ and $\mathbf{C O N T}_{\mathbf{x}}$ (on average: $r=0.91, p<0.001$, Additional file 4: Figure S4A), and $\mathbf{M V}_{\mathbf{L}}, \mathbf{A}, \mathbf{A}_{\mathbf{L}}-$ pos, $\mathbf{A}_{\mathbf{L}}-\mathbf{n e g}$ with $\mathbf{M V}$ (on average: $r=0.94$, $p<0.001)$.

The model was able to reproduce both values and trends (between $T_{0}$ and $T_{1}$ ) in the vast majority of the Evaluation parameters (Additional file 3: Figure S3 comparison between gray and colored bars). In particular, movement speed increased, as already showed by the Model-based parameters and further supported by the significant increase in $\mathbf{M V}_{\mathbf{L}}, \mathbf{A}, \mathbf{A}_{\mathbf{L}}-\mathbf{p o s}$, and $\mathbf{A}_{\mathbf{L}}-\mathbf{n e g}$ for real and simulated trajectories both for sub-acute and chronic patients $(p<0.05)$. Amplitude of transversal peaks $\left(\mathbf{A}_{\mathbf{N}}-\right.$ pos and $\left.\mathbf{A}_{\mathbf{N}}-\mathbf{n e g}\right)$, instead, significantly increased for sub-acute patients and decreased for chronic patients $(p<0.05)$. The simulations also reproduced a raise in the movement smoothness, which was accompanied by a significant reduction of positive and negative peaks both for transversal and longitudinal velocities (nPK $\mathbf{L}_{\mathrm{L}}-$ pos, $\mathbf{n P K}_{\mathrm{L}}-\mathbf{n e g}, \mathbf{n P K}_{\mathbf{N}}-$ pos, and $\mathbf{n P K}_{\mathbf{N}}-\mathbf{n e g}$ ) $(p<0.05)$. Finally, MD significantly decreased between $T_{0}$ and $T_{1}$ for both patient groups $(p<0.05)$. This decrease could be explained by a translation of the movements toward the longitudinal direction as supported from the increment of $\mathbf{C O N T}_{\mathbf{L}}$-amp and the reduction of $\mathbf{C O N T}_{\mathbf{N}^{-}}$-amp.

\section{Different aspects of motor recovery highlighted by the model-based parameters}

When looking at internal correlations within the Modelbased parameters (Fig. 5a) we observed that nPK was highly correlated with $\mathbf{t}(r=0.94, p<0.001)$, in agreement with [10], suggesting that submovement blending was strictly intermingled with movement duration shortening. Other parameters, computed on transversal and longitudinal velocities, such as $\langle\sigma\rangle$, ratio-nPK, ratio$\mathbf{a m p}_{\mathbf{L}}$, and ratio-amp $\mathbf{p}_{\mathbf{N}}$, instead, were not correlated with the others (on average: $r=0.24, p>0.05$ ), suggesting that they were likely representing different aspects of motor improvements.

When FA was used to inspect for redundancy among the Model-based parameters and to investigate more in detail the different factors involved in motor recovery and particularly which variables measured similar aspects of motor improvement, three main factors were found, explaining together $70 \%(52 \%, 12 \%$, and $6 \%$, respectively) of the total variance (Fig. 5b). The first major factor decreased during the rehabilitation, was related to variables mainly accounting for movement inefficiency (nPK, $\mathbf{C O N T}_{\mathbf{L}}, \mathbf{C O N T}_{\mathbf{N}}$, and t), and correlated with FMA score $(r=0.60, p=0.002$, Fig. $5 \mathrm{c})$. The second factor increased during the rehabilitation, was related to $\mathbf{M V}$ and $\mathbf{M V}_{\mathbf{N}}$ (movement speed), and correlated with MAS score for the shoulder $(r=0.68, p<0.001)$. Finally, the third factor correlated with FMA score $(r=0.50$, $p=0.015)$ and was described by ratio- $\mathbf{a m p}_{\mathbf{N}}$, which relates to movement inaccuracy as detailed by the high correlation with $\mathrm{MD}(r=0.68, p<0.001)$.

Interestingly the temporal dynamics during the therapy was different for the three factors (Fig. 5d) suggesting different central and/or peripheral mechanisms concurrently involved in patients' motor recovery, each with a specific temporal scale. In particular, for subacute patients the first and the third factors showed a double-exponential decay, with a comparable fast component $(\tau \approx 0.5$ and 0.3 , respectively), which was even faster than the increase of movement speed ( $\tau \approx 2.5$ sessions). The slow component, instead, had a larger decay time constant for movement inaccuracy ( $\tau \approx 10$ sessions) than for inefficiency ( $\tau \approx 5.6$ sessions), i.e., inaccuracy was the slowest to reach a plateau in its temporal evolution during rehabilitation. The best fit for the time courses of the three factors for chronic patients were straight lines. The slopes values revealed a mild decrease for movement inefficiency $(\mathrm{m} \approx-0.005)$ over rehabilitation, and a more evident increase for movement speed $(\mathrm{m} \approx 0.03)$ and decrease for inaccuracy $(\mathrm{m} \approx-0.05)$.

\section{Discussion}

In this study we introduced a computational model of trajectory generation to identify a set of ten variables, the Model-based parameters, which allow for an exhaustive kinematic characterization of upper-limb planar movements performed by post-stroke hemiparetic subjects during robot-based training. We then demonstrated that these parameters are informative of three main underlying (and unobserved) recovery factors (movement inefficiency, speed, and inaccuracy), each one characterized by a specific time evolution, and, thus, putatively revealing a different central and/or peripheral mechanism of motor restoration.

\section{A computational model of post-stroke subjects' trajectories}

Previous computational models of trajectory generation in healthy adult subjects include motor control strategies 


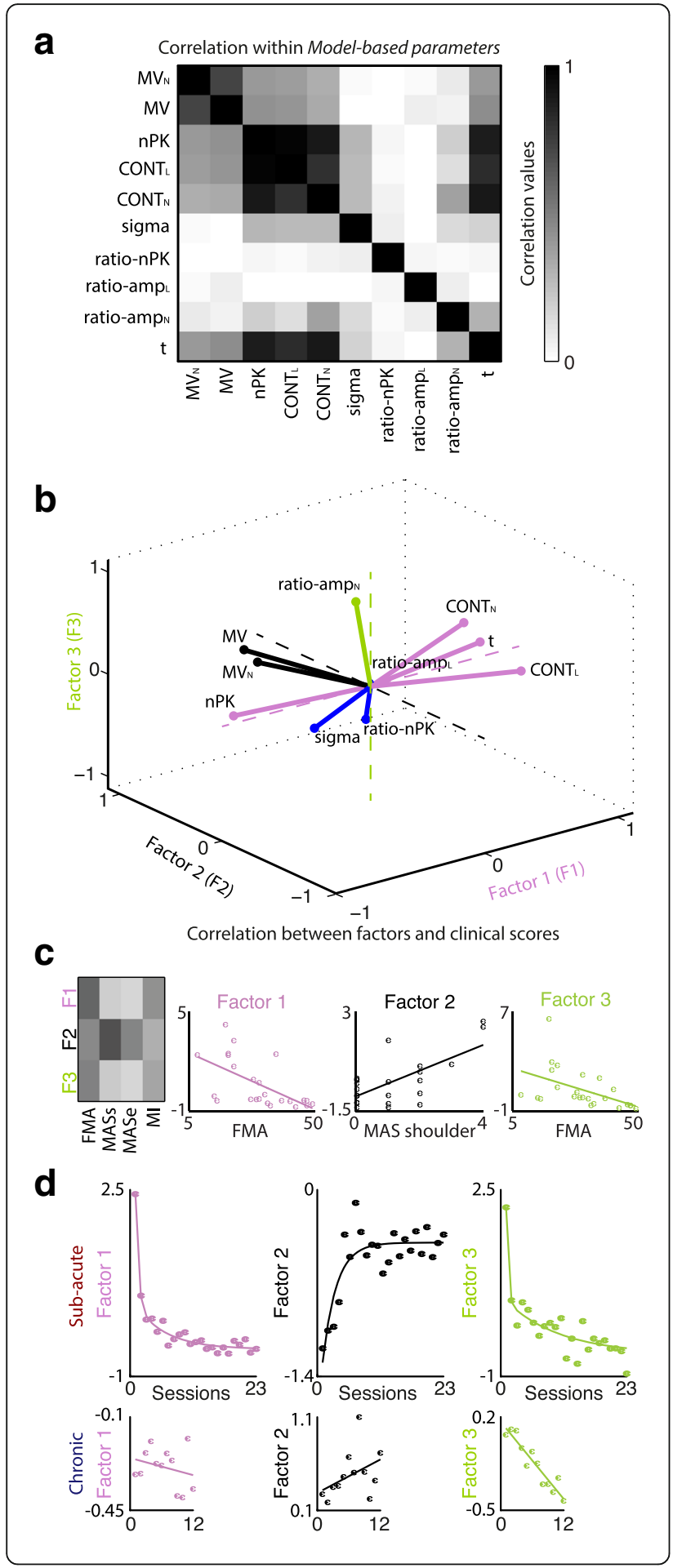

Fig. 5 Factorial analysis of Model-based parameters. a Correlation matrix for the Model-based parameters. $\mathbf{b}$ Three-dimensional factor representation of the Model-based parameters. Pink, black, and green lines code the parameters associated with factor 1, factor 2 , and factor 3, respectively. Blue lines code the parameters that are "shared" across factors. c Right: correlation matrix between factors and clinical scores. The colorbar is the same of panel (a). MASs and MASe abbreviate MAS shoulder and MAS elbow, respectively. Left: Scatter plots of the correlation between the three main factors and the clinical scores. $\mathbf{d}$ Temporal evolution of the three factors for sub-acute (top row) and chronic patients (bottom row). Each point represents the value of the factor for each session averaged across patients. Thick lines represent the results of the fitting of experimental data (i.e., linear, exponential or double exponential). Y-axis scales are different for each factor and patients group

explained by minimum variance [33] minimum jerk [34], minimum torque change [35], and optimal feedback control [36]. However, motor control strategies are often modified by brain insults $[37,38]$ reflecting the need for tampered computational models. Therefore, in order to generate post-stroke trajectories, we designed a model based on the well-known mechanism of submovement blending [3]. Indeed, in our model, tangential speed profiles of point-to-point movements were assumed to be a sum of submovement curves with bell-shaped Gaussian profiles, i.e., the tangential speed profile was composed of a finite sum of submovements with similar shapes but dilated in duration, translated in time, and modulated in amplitude. A previous work has demonstrated that submovement speed profiles from stroke patients can be appropriately approximated by nearly symmetric $\beta$ functions, and that the differences with Gaussian curves were rather minimal [24]. We, therefore, used Gaussian curves for tangential speed and extended this assumption also to transversal and longitudinal velocity profiles. These results would require further experimental validation on a larger population of stroke subjects.

The simulated trajectories generated by our computational model were significantly similar to the real trajectories from post-stroke patients and were able to reproduce the trends in the Evaluation parameters. These results demonstrated the capability of the Model-based parameters to well characterize the kinematics of patients' movements and to discriminate between different impairment levels. Additionally, small differences were found between real and simulated angular excursions of limb joints, indicating that impairments at the joint level are influenced by the number and amplitude of peaks in both the transversal and longitudinal speed components. However, the angular excursions at the elbow were better reproduced than those at the shoulder (5\% versus $19 \%$ ), probably because of the two-link model limitations to reconstruct shoulder movements normally characterized by three degrees of freedom [25]. 
Our computational model is currently limited to simulate trajectories of movements autonomously performed by post-stroke subjects which could restrict the applicability of the proposed framework to patients with moderate to mild impairments. Indeed, patients with more severe functional damages most often require robot assistance to accomplish the task [39]. Future works are recommended to address this issue, and to extend the model to other volitional arm movements and assistive devices, such as exoskeletons [40-43]. Indeed, exoskeletons offer several advantages over planar manipulandum because they enlarge the task space to three dimensions following the arm in its natural workspace with no restrictions [44]. Therefore, an extension of our model to three-dimensional trajectories would be of interest for further applications.

Interestingly this model could represent an element of a larger framework for testing new solutions for the reinforcement and the personalization of the therapy [45]. Simulated trajectories could be used to assess whether the patient's movements follow the expected improvement, thus prompting 'real-time' clinical actions. Indeed, the values of Model-based parameters computed from real trajectories at $T_{O}$ and the estimates of motor improvement trends along the rehabilitation training could be fed to the model and used to generate reference trajectories for a given rehabilitation session. Upon comparing these trajectories with the real ones from the patient, actions for task assistance could be immediately triggered, and training refinement could be rightly planned and implemented.

\section{Model-based kinematic parameters of point-to-point trajectories}

Four variables used by the model: $\mathbf{M V} \mathbf{n P K},\langle\boldsymbol{\sigma}\rangle$, and $\mathbf{t}$, are well-known parameters proposed by a number of previous studies to characterize motor improvements in post-stroke subjects [5, 39, 46, 47]. They are extracted from the tangential speed profile and they neglect aspects of motor improvement related to end-point accuracy and directional errors. For this reason, they are often complemented by other measures to achieve a more exhaustive description of motor improvement. These additional parameters (e.g., the "mean distance from theoretical path", the "trajectory straightness", etc. [47, 48]), however, require a priori assumption that point-to-point movements from fully recovered patients, as well as from healthy subjects, are straight, which might be ultimately wrong [49]. Instead, here, we proposed new variables computed from the transversal and longitudinal speed profiles that do not require $a$ priori assumptions on movement straightness.

Among these new parameters $\mathbf{M V}_{\mathbf{N}}, \mathbf{C O N T}_{\mathbf{N}}$, and ratio$\mathbf{a m p}_{\mathbf{N}}$ were initially considered putative parameters to assess movement accuracy, because they were computed from submovements transversal to the theoretical path. However, only ratio-amp $\mathbf{p}_{\mathbf{N}}$ was highly correlated with $\mathbf{M D}$, a well-known measure of movement accuracy [39, 47], whereas $\mathbf{C O N T}_{\mathbf{N}}$ turned out to be negatively correlated with $\mathbf{n P K}$, and $\mathbf{M V}_{\mathbf{N}}$ positively correlated with $\mathbf{M V}$. The latter was rather unexpected and counterintuitive, because a reduction of average movement speed in the transversal direction was expected, based on the well-documented tendency of post-stroke trajectories to become straighter with training [49]. Instead, $\mathbf{M} \mathbf{V}_{\mathbf{N}}$ increased in parallel with MV, presumably reflecting improved general muscle tone as also demonstrated by the correlation with the MAS score for the shoulder $(r=0.50, p=0.002$, see Additional file 4: Figure S4B) [11]. The increase of accuracy, instead, was probably obtained by reducing the speed of transversal submovements with respect to longitudinal movements, a complex strategy most likely planned and realized by more central mechanisms involved in motor recovery.

Taken together these new insights significantly contribute to expand the current theory of post-stroke motor recovery based on discrete submovements blending [3].

\section{Kinematic markers of motor recovery}

In our cohort of patients the evaluation of the clinical scales at time $T_{O}$ and $T_{1}$ showed that robot-aided therapy led to a reduction of impairment in the hemiparetic limb. This was paralleled by improvements in point-topoint upper limb movements which became progressively similar to those of healthy subjects. We here have to acknowledge the limited sample size of the two patient groups that reduces the strength of the statistical findings. However, the study in which the patients were enrolled was designed as a pilot study [16] with the straight restriction to recruit patients with absence of bilateral impairments.

The application of Factorial Analysis to the Model-based parameters showed that the latter were redundant to some extent and informative of three main recovery factors.

The first factor named movement inefficiency, was mainly related to variables describing temporal efficiency (movement duration) and movement smoothness. This result confirms and reinforces the quantitative analysis made by Alt Murphy et al. [10] and it advises a reinterpretation of previous disentanglement between these two variables into two different aspects of movement impairments: paresis (i.e., the decreased ability to volitionally modulate motor units activation [13]) and abnormal muscle tone [11]. Moreover, this result suggests that submovement blending, which caused improved movement smoothness, was also one of the main causes of movement duration shortening. The second factor, movement speed, was intimately related to muscle tone [11], whereas the third factor (i.e., movement inaccuracy) 
was mainly associated to the decrease of position errors along the primary axis of movement and, thus, to the loss of fractionated movement [11]. The decrease of the fast component of the first and third factors was slightly faster than the increase of movement speed. The slow component, instead, was slower for both factors, particularly for movement inaccuracy, which was the slowest to reach a plateau in its temporal evolution during rehabilitation. Similar temporal evolutions of the three factors during the rehabilitation process were visible also for chronic patients. However, the best fits for chronic patients were straight lines. Different evolution of the recovery factors could be already expected from the time course of the Model-based parameters, which had a linear and exponential evolution for chronic and sub-acute patients, respectively (Fig. 4), and could be related to a more rapid and generalized improvement in early poststroke period, as suggested in [50], and to a different treatment length.

The importance of the Model-based parameters as markers of motor recovery was highlighted also from the correlation with the different clinical scores (see Additional file 4: Figure $\mathrm{S} 4 \mathrm{~B}$ and $5 \mathrm{c}$ ). In particular the Model-based parameters correlated with the wellaccepted FMA score and the MAS score. Moreover, as expected from the assessment of the clinical scales, the first and the third factors correlated significantly with the FMA score, while the second factor had a significant correlation with the MAS shoulder score. Indeed, the FMA scale evaluates complex active movements that require the activation of several joints paralleling motor efficiency. The MAS scale, instead, evaluates the resistance to passive single-joint movements and it could, thus, relate to the movement speed, which decreases with spasticity.

Conclusively the characterization of longitudinal and transversal velocities seems to be essential in clinical application for an exhaustive description of the patients' motor impairments. The metrics proposed here could be used to complement therapists with immediate measures of motor performance [6] and to design more effective rehabilitation protocols targeted to differentially reduce gap performances in the two orthogonal directions. Interestingly, the existence of correlations among the Model-based parameters, and particularly among the parameters related to movement inefficiency, and the fact that only three main factors of motor improvement were found by FA point to the possibility to further reduce the number of kinematic variables needed for a computational model to simulate stroke-like trajectories. In this regard, a further study in a larger cohort of patients will be necessary to establish mathematical relationships among the Model-based parameters, in order to further reduce their redundancy.

\section{Conclusions}

In this study, we defined a set of kinematic parameters for the characterization of upper limb movements performed by post-stroke hemiparetic subjects during robot-based training. Despite the considerable development of robot-assisted therapy in clinical practice and the various kinematic variables suggested so far, there is still no a general consensus on which parameters to use to evaluate the movement performance. The metrics proposed here are based on a model-driven approach, rather than on specific study hypotheses or on sought relationships with clinical scales. We demonstrated that i) they capture relevant kinematic information to assess the quality of reaching trajectories; ii) they are manageable, i.e., they do not necessarily require data reduction techniques to extract information about movement performance from a large dataset of computed parameters; iii) they reveal diverse factors of kinematic improvement over time, which are informative of different central and/or peripheral mechanisms of motor recovery. By monitoring how these factors change over time at the individual level may provide a new tool to help physiotherapist to take decisions regarding treatment planning.

\section{Additional files}

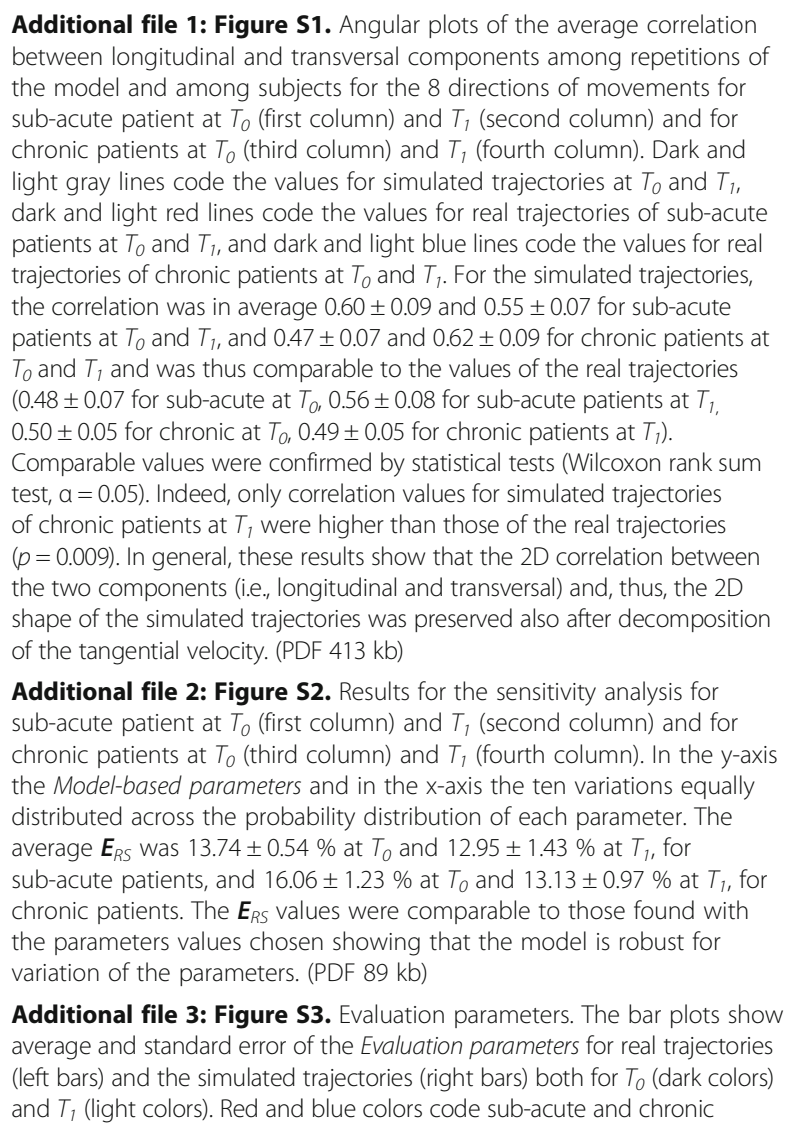


patients. Asterisks ${ }^{*}$ ) indicate significant differences (Wilcoxon signed-rank test, $p<0.05$ ) between $T_{0}$ and $T_{1}$ for sub-acute (red), chronic (blue), and modeled trajectories (grey). (PDF $273 \mathrm{~kb}$ )

Additional file 4: Figure S4. A) Correlation matrix for Model-based parameters and Evaluation parameters. B) Correlation matrix for Model-based parameters and clinical scores. (PDF $188 \mathrm{~kb}$ )

\section{Acknowledgements}

We would like to thank all the volunteers enrolled in the study.

\section{Funding}

This work was partially supported by the Wyss Center for Bio and Neuroengineering, the MIRROR Project (Novel Approaches for Robot-Mediated Neuro-Rehabilitation) funded by a local Bank Foundation (Fondazione MPS), and by the RISDOM Project by the Government of Tuscany (Italy) and the Municipality of Peccioli (Italy). This work was supported by the RONDA Project, funded by Regione Toscana PAR FAS 2007-2013.

\section{Availability of data and materials}

Since the data used in this study were collected in a clinical trial including patients, the data will not be shared. The Matlab code of the model will be available upon request to the corresponding authors of this study.

\section{Authors' contributions}

AP designed the model, analyzed data, and wrote the paper; EP designed the model, analyzed data, and wrote the paper; PT carried out experiments, and wrote the paper; BC carried out experiments, and wrote the paper; FP carried out experiments, and wrote the paper; SM designed the model, and wrote the paper. All authors read and approved the final manuscript.

\section{Competing interests}

The authors declare that they have no competing interests.

\section{Consent for publication}

All participants signed an informed consent to the use of all data collected during the experiment in scientific publications.

\section{Ethics approval and consent to participate}

All participants signed an informed consent in accordance with the policies about trials with human subjects before starting experimental and rehabilitation sessions. The protocol was approved by the Local Ethical Committee (Comitato Etico Azienda Ospedaliera Universitaria di Pisa. Reference $N^{\circ}:$ 2754, year 2009).

\section{Author details}

'The BioRobotics Institute, Scuola Superiore Sant'Anna, Viale R. Piaggio 34, 56025 Pontedera, Pisa, Italy. ${ }^{2}$ Bertarelli Foundation Chair in Translational Neuroengineering, Center for Neuroprosthetics and Institute of Bioengineering, School of Engineering, École Polytechnique Fédérale de Lausanne (EPFL), Lausanne, Switzerland. ${ }^{3}$ Centre of Space Bio-medicine, University of Rome Tor Vergata, Rome, Italy. ${ }^{4}$ Laboratory of Neuromotor Physiology, IRCCS Santa Lucia Foundation, Rome, Italy. ${ }^{5}$ Rehabilitation Department Versilia Hospital, AUSL 12, Viareggio, Italy. ${ }^{6}$ Bioengineering Rehabilitation Laboratory, Auxilium Vitae Rehabilitation Centre, Volterra, Italy.

Received: 6 February 2016 Accepted: 26 August 2016

Published online: 08 September 2016

\section{References}

1. Gresham GE, Duncan PW, Stason WB. Post-stroke rehabilitation. Darby: DIANE Publishing; 1997.

2. Donnan GA, Fisher M, Macleod M, Davis SM. Stroke. Lancet. 2008;371:1612-23.

3. Rohrer B, Fasoli S, Krebs HI, Hughes R, Volpe B, Frontera WR, Stein J, Hogan N. Movement smoothness changes during stroke recovery. J Neurosci. 2002; 22:8297-304.

4. Patton J, Small SL, Zev Rymer W. Functional restoration for the stroke survivor: informing the efforts of engineers. Top Stroke Rehabil. 2008;15:521-41.

5. Krebs HI, Krams M, Agrafiotis DK, DiBernardo A, Chavez JC, Littman GS, Yang E, Byttebier G, Dipietro L, Rykman A. Robotic measurement of arm movements after stroke establishes biomarkers of motor recovery. Stroke. 2014;45:200-4.

6. Bosecker C, Dipietro L, Volpe B, Krebs HI. Kinematic robot-based evaluation scales and clinical counterparts to measure upper limb motor performance in patients with chronic stroke, Neurorehabilitation and neural repair. 2009.

7. Sanford J, Moreland J, Swanson LR, Stratford PW, Gowland C. Reliability of the fugl-meyer assessment for testing motor performance in patients following stroke. Phys Ther. 1993;73:447-54.

8. Krebs HI, Volpe BT, Ferraro M, Fasoli S, Palazzolo J, Rohrer B, Edelstein L, Hogan N. Robot-aided neurorehabilitation: from evidence-based to sciencebased rehabilitation. Top Stroke Rehabil. 2002;8:54-70.

9. Gregson JM, Leathley MJ, Moore AP, Smith TL, Sharma AK, Watkins CL. Reliability of measurements of muscle tone and muscle power in stroke patients. Age Ageing. 2000;29:223-8.

10. Murphy MA, Willén C, Sunnerhagen KS. Kinematic variables quantifying upper-extremity performance after stroke during reaching and drinking from a glass. Neurorehabil Neural Repair. 2011;25:71-80.

11. Nordin N, Xie SQ, Wünsche B. Assessment of movement quality in robot-assisted upper limb rehabilitation after stroke: a review. J Neuroeng Rehabil. 2014;11:137.

12. Kitago T, Marshall RS. Strategies for early stroke recovery: what lies ahead? Curr Treat Options Cardiovasc Med. 2015;17:1-10.

13. Lang CE, Bland MD, Bailey RR, Schaefer SY, Birkenmeier RL. Assessment of upper extremity impairment, function, and activity after stroke: foundations for clinical decision making. J Hand Ther. 2013;26:104-15.

14. Fugl-Meyer AR. Post-stroke hemiplegia assessment of physical properties. Scand J Rehabil Med Suppl. 1980;7:85.

15. Fugl-Meyer AR, Jääskö L, Leyman I, Olsson S, Steglind S. The post-stroke hemiplegic patient. 1. a method for evaluation of physical performance. Scand J Rehabil Med. 1974;7:13-31.

16. Tropea P, Monaco V, Coscia M, Posteraro F, Micera S. Effects of early and intensive neuro-rehabilitative treatment on muscle synergies in acute poststroke patients: a pilot study. J Neuroeng Rehabil. 2013;10:103.

17. Cesqui B, Macrì G, Dario P, Micera S. Characterization of age-related modifications of upper limb motor control strategies in a new dynamic environment. J Neuroeng Rehabil. 2008;5:31.

18. Krebs HI, Hogan N, Aisen ML, Volpe BT. Robot-aided neurorehabilitation. IEEE Trans Rehabil Eng. 1998;6:75-87.

19. Bohannon RW, Smith MB. Interrater reliability of a modified ashworth scale of muscle spasticity. Phys Ther. 1987;67:206-7.

20. Collin C, Wade D. Assessing motor impairment after stroke: a pilot reliability study. J Neurol Neurosurg Psychiatry. 1990;53:576-9.

21. Burdet $E$, Milner TE. Quantization of human motions and learning of accurate movements. Biol Cybern. 1998;78:307-18.

22. Balasubramanian S, Melendez-Calderon A, Burdet E. A robust and sensitive metric for quantifying movement smoothness. Biomed Eng IEEE Trans. 2012;59:2126-36.

23. Flash $\mathrm{T}, \mathrm{Hogan} \mathrm{N}$. The coordination of arm movements: an experimentally confirmed mathematical model. J Neurosci. 1985;5:1688-703.

24. Krebs HI, Aisen ML, Volpe BT, Hogan N. Quantization of continuous arm movements in humans with brain injury. Proc Natl Acad Sci. 1999;96:4645-9.

25. Dipietro L, Krebs HI, Fasoli SE, Volpe BT, Stein J, Bever C, Hogan N. Changing motor synergies in chronic stroke. J Neurophysiol. 2007;98:757-68.

26. Ellis MD, Sukal T, DeMott T, Dewald JP. Augmenting clinical evaluation of hemiparetic arm movement with a laboratory-based quantitative measurement of kinematics as a function of limb loading. Neurorehabil Neural Repair. 2008;22:321-9.

27. Ellis MD, Sukal-Moulton T, Dewald JP. Progressive shoulder abduction loading is a crucial element of arm rehabilitation in chronic stroke. Neurorehabil Neural Repair. 2009;23:862-9.

28. Sukal TM, Ellis MD, Dewald JP. Shoulder abduction-induced reductions in reaching work area following hemiparetic stroke: neuroscientific implications. Exp Brain Res. 2007;183:215-23.

29. Diffrient N, Tilley AR, Bardagjy JC. Humanscale 1/2/3: a portfolio of information. Cambridge: MIT Press; 1974.

30. Osborne JW, Costello AB. Best practices in exploratory factor analysis: four recommendations for getting the most from your analysis. Pan-Pacific Manag Rev. 2009;12:131-46.

31. Box J: Reinsel, editor. Time Series Analysis, Forecasting and Control. Englewood Clifs. NJ: Prentice Hall; 1994.

32. Wei X-J, Tong $\mathrm{K}-\mathrm{y}$, Hu X-I. The responsiveness and correlation between fuglmeyer assessment, motor status scale, and the action research Arm test in 
chronic stroke with upper-extremity rehabilitation robotic training. Int J Rehabil Res. 2011;34:349-56.

33. Harris CM, Wolpert DM. Signal-dependent noise determines motor planning. Nature. 1998:394:780-4.

34. Hogan N. An organizing principle for a class of voluntary movements. J 727 Neurosci. 1984:4:2745-54

35. Uno Y, Kawato M, Suzuki R. Formation and control of optimal trajectory in human multijoint arm movement. Minimum torque-change model. Biol Cybern. 1989;61:89-101.

36. Todorov E, Jordan MI. Optimal feedback control as a theory of motor coordination. Nat Neurosci. 2002;5:1226-35.

37. Cheung VC, Piron L, Agostini M, Silvoni S, Turolla A, Bizzi E. Stability of muscle synergies for voluntary actions after cortical stroke in humans. Proc Natl Acad Sci U S A. 2009;106:19563-8.

38. Cheung VC, Turolla A, Agostini M, Silvoni S, Bennis C, Kasi P, Paganoni S, Bonato P, Bizzi E. Muscle synergy patterns as physiological markers of motor cortical damage. Proc Natl Acad Sci U S A. 2012;109:14652-6.

39. Colombo R, Pisano F, Micera S, Mazzone A, Delconte C, Carrozza CM, Dario P, Minuco G. Robotic techniques for upper limb evaluation and rehabilitation of stroke patients. Neural Syst Rehabil Eng IEEE Trans. 2005;13:311-24.

40. Nef T, Guidali M, Riener R. ARMin III-arm therapy exoskeleton with an ergonomic shoulder actuation. Appl Bionics Biomech. 2009;6:127-42.

41. Carignan C, Liszka M, Roderick S. Design of an arm exoskeleton with scapula motion for shoulder rehabilitation. In Advanced Robotics, 2005 ICAR'05 Proceedings, 12th International Conference on. IEEE; 2005. 524-531.

42. Perry JC, Rosen J Design of a 7 degree-of-freedom upper-limb powered exoskeleton. In Biomedical Robotics and Biomechatronics, 2006 BioRob 2006 The First IEEE/RAS-EMBS International Conference on. IEEE; 2006. 805-810.

43. Pirondini E, Coscia M, Marcheschi S, Roas G, Salsedo F, Frisoli A, Bergamasco M, Micera S. Evaluation of the effects of the Arm light exoskeleton on movement execution and muscle activities: a pilot study on healthy subjects. J Neuroeng Rehabil. 2016;13:1.

44. Milot M-H, Spencer SJ, Chan V, Allington JP, Klein J, Chou C, Bobrow JE, Cramer SC, Reinkensmeyer DJ. A crossover pilot study evaluating the functional outcomes of two different types of robotic movement training in chronic stroke survivors using the arm exoskeleton BONES. J Neuroeng Rehabil. 2013;10:112.

45. Casadio M, Tamagnone I, Summa S, Sanguineti V. Neuromotor recovery from stroke: computational models at central, functional, and muscle synergy level. Front Comput Neurosci. 2013;7:97.

46. Colombo R, Pisano F, Micera S, Mazzone A, Delconte C, Carrozza MC, Dario P, Minuco G. Assessing mechanisms of recovery during robot-aided neurorehabilitation of the upper limb. Neurorehabil Neural Repair. 2008;22:50-63.

47. Panarese A, Colombo R, Sterpi I, Pisano F, Micera S. Tracking motor improvement at the subtask level during robot-aided neurorehabilitation of stroke patients. Neurorehabil Neural Repair. 2012;26:822-33.

48. Cirstea MC, Levin MF. Compensatory strategies for reaching in stroke. Brain. 2000;123(Pt 5):940-53.

49. Wisneski KJ, Johnson MJ. Quantifying kinematics of purposeful movements to real, imagined, or absent functional objects: Implications for modelling trajectories for robot-assisted ADL tasks. J Neuroeng Rehabil. 2007;4:7.

50. Krakauer JW, Carmichael ST, Corbett D, Wittenberg GF. Getting neurorehabilitation right what can be learned from animal models? Neurorehabil Neural Repair. 2012;26:923-31.

\section{Submit your next manuscript to BioMed Centra and we will help you at every step:}

- We accept pre-submission inquiries

- Our selector tool helps you to find the most relevant journal

- We provide round the clock customer support

- Convenient online submission

- Thorough peer review

- Inclusion in PubMed and all major indexing services

- Maximum visibility for your research

Submit your manuscript at www.biomedcentral.com/submit

) Biomed Central 